\title{
Erlangian Approximation to Finite Time Ruin Probabilities in Perturbed Risk Models
}

\author{
Kaiqi Yu, David A. Stanford, Jiandong Ren \\ Department of Statistical \& Actuarial Sciences, \\ The University of Western Ontario, London, Ont., Canada N6A5B7
}

March 4, 2008

\begin{abstract}
In this work-in-progress, we consider perturbed risk processes that have an underlying Markov structure, including Markovian risk processes, and Sparre-Andersen risk processes when both inter claim times and claim sizes are phase-type. We apply the Erlangization method to this risk process in order to obtain an accurate approximation of the finite time ruin probability. In addition, we recognize a repeating structure in the probability matrices we work with. Numerical examples illustrate the use of the model.
\end{abstract}

Keywords: Perturbed risk processes, finite-time ruin probability, phase-type distribution, fluid flow models, Erlangization.

\section{Introduction}

This work-in-progress considers the aggregate loss model

$$
S_{t}=\sum_{k=1}^{N_{t}} Z_{k}-c t-\sigma \xi_{t},
$$

where $c>0$ is the premium received per time unit, $\left\{Z_{i} ; i=1,2, \ldots\right\}$ are independent non-negative claim random variables, the process $\left\{N_{t}\right\}_{t \geq 0}$ counts the number of claims up to time $t, \sigma>0$, and $\left\{\xi_{t}\right\}_{t \geq 0}$ is a standard Brownian motion. In the present paper, we assume that the claim amounts $Z_{i} ; i=1,2, \ldots$ are phase-type distributed. In addition, in the development which follows, we will let the other parameters of the model (the inter-claim times, the premium rate, and the variability parameter $\sigma$ ) depend upon the state of an underlying Markov chain.

The time of ruin is

$$
\tau=\tau(u)=\inf \left\{t>0: S_{t} \geq u\right\}
$$


where $u \geq 0$ is the initial reserve with the understanding that $\tau=\infty$ if ruin never occurs. The infinite horizon ruin probability is $\psi(u)=\mathbf{P}(\tau(u)<\infty)$ and the probability of ruin before time $T$ is

$$
\psi(u, T)=\mathbf{P}(\tau(u) \leq T) .
$$

The ultimate ruin probability $\psi(u)$ for this model has been studied extensively; see for example, Dufrense \& Gerber [10] and Tsai \& Willmot [19] for the classical case, Li \& Garrido [11] for the case where the interclaim times following a generalized Erlang distribution, and Lu \& Tsai [12] for the case where $\left\{N_{t}\right\}_{t \geq 0}$ is a Markov modulated Poisson process. The methods used are based on constructing and solving defective renewal equations, yielding formulas for $\psi(u)$ or related quantities.

The exact determination of the finite time ruin probability is considered much harder than that of its infinite-time counterpart. Efforts have been made to obtain $\psi(u, T)$ in various non-perturbed risk models for some time, along a variety of lines. Thorin \& Wikstad ([17] and [18]) used a root finding method for selected SparreAndersen models. Drekic \& Willmot [9] obtained $\psi(u, T)$ in the classical risk model for the case of exponentially-distributed claim sizes, based on evaluating Bessel functions. By discretising time, Dickson \& Waters [8] obtained a recursive formula at successive time instants for $\psi(u, T)$ for classical models. Stanford \& Stroiński [15] and Stanford et al. [16] tried to avoid discretising time for $\psi(u, T)$ in classical models and a few Sparre-Andersen models, respectively. Instead, they embedded the recursion at claim epochs. Asmussen, Avram \& Usabel [4] developed the socalled "Erlangization" technique to approximate the finite time ruin probability for the classical risk model with phase type claim sizes, while Stanford et al. [14] applied Erlangization to the Sparre-Andersen and stationary renewal risk model cases.

This paper applies Erlangization to the perturbed risk model defined by (1) to get an accurate approximation of the finite time ruin probability. In addition, the approximation is asymptotically exact as the order of the Erlang increases. The present work, alongside a Martingale approach of Badescu \& Breuer [5], are the first attempts we know of to either compute or approximate finite time ruin probabilities in the perturbed risk model.

The main reason why it is typically so much harder to determine $\psi(u, T)$ than $\psi(u)$ is precisely due to the finite time horizon, which makes it hard to establish recursions over time. Erlangization circumvents this problem by replacing fixed time intervals with random, open-ended intervals with the same means as the finite time periods of interest. In so doing, the renewal structure is regained. The simplest such random interval is of course an exponential distribution with mean $T$. The sequence of Erlang distributions $\operatorname{Er}(L), L=1,2, \ldots$, with a common mean $T$ converges to a point mass at $T$ of probability one as $L \rightarrow \infty$.

In order to exploit the solution method we propose below, it is necessary to associate the risk model $\left\{S_{t}\right\}_{t \geq 0}$ defined in (1) with a sample-path-equivalent Markovian fluid flow. Markovian fluid flows have rates of increase and decrease based on the underlying state of the associated continuous-time Markov chain $\left\{J_{t}\right\}_{t \geq 0}$. For risk models that can be accommodated in this way, we construct a composite process 
that keeps track of both the state of $\left\{J_{t}\right\}_{t \geq 0}$ as well as the Erlang stage. Then by exploiting established results for Markovian fluid flows, we are able to determine our accurate approximation to $\psi(u, T)$. In the full paper of the same name that has been completed since the Dagstuhl proceedings, we have been able to develop a more efficient procedure by recognizing a repeating structure in the probability matrices we work with.

The remainder of this work-in-progress is organized as follows. Following the mathematical preliminaries and an illustrative example, Section 3 recaps the essential elements we need from Asmussen [1]. Section 4 explains Erlangization and the construction of our composite process. Section 5 states the resulting form of the ruin probability and its components due to drift and claims. Section 6 describes the repetitive block structure of matrices involved, in order to devise a more efficient algorithm. Numerical examples are presented in section 7 .

\section{Mathematical Preliminaries}

As introduced by Asmussen [2], given any sample path for the risk process (see top part of Figure 1 below), a sample-path-equivalent fluid flow the lower part of Figure 1) can be constructed as follows. When interclaim times and claim sizes have a Markovian structure, we may associate the aggregate loss process $\left\{S_{t}\right\}_{t \geq 0}$ with an equivalent Markovian fluid flow process $\left\{\left(J_{t}, \tilde{S}_{t}\right)\right\}_{t \geq 0}$. The process $\left\{J_{t}\right\}_{t \geq 0}$ keeps track of the underlying phases of the Markov chain affecting the rate of fluid flow at time $t$, while $\left\{\tilde{S}_{t}\right\}_{t \geq 0}$ indicates the level of fluid at time $t$. This is not a narrow restriction because as will be shown in next section, a wide range of risk processes have such structure. The simplest mechanism to establish a one-to-one correspondence between the sample path of the perturbed risk process and that of the partially-perturbed fluid flow is to choose positive rate $r=1$ for the increasing phases in the fluid level (see Figure 1). By doing so, the amount of elapsed "artificial" time equals the claim size, as does the amount of rise. During these periods of artificial time, $\left\{J_{t}\right\}_{t \geq 0}$ keeps track of the phase of the phase-distributed claim size. Between claims, the risk process's sample path is replicated in the fluid process.

We partition the phase set $E$ of the Markov jump process $\left\{J_{t}\right\}_{t \geq 0}$ into two sets $E_{\sigma}$ and $E_{+}$as follows:

- $E_{\sigma}$ corresponds to the states during the time intervals between claims, where $\tilde{S}_{t}$ is subject to the diffusion component;

- $E_{+}$corresponds to the the states representing the claims, which evolve over artificial time. In these states, $\tilde{S}_{t}$ simply increases at unit rate and is not subject to the diffusion component.

The infinitesimal generator of process $J(t)$ then has the form

$$
\boldsymbol{\Lambda}=\left(\begin{array}{ll}
\Lambda^{(\sigma \sigma)} & \boldsymbol{\Lambda}^{(\sigma+)} \\
\Lambda^{(+\sigma)} & \Lambda^{(++)}
\end{array}\right)
$$



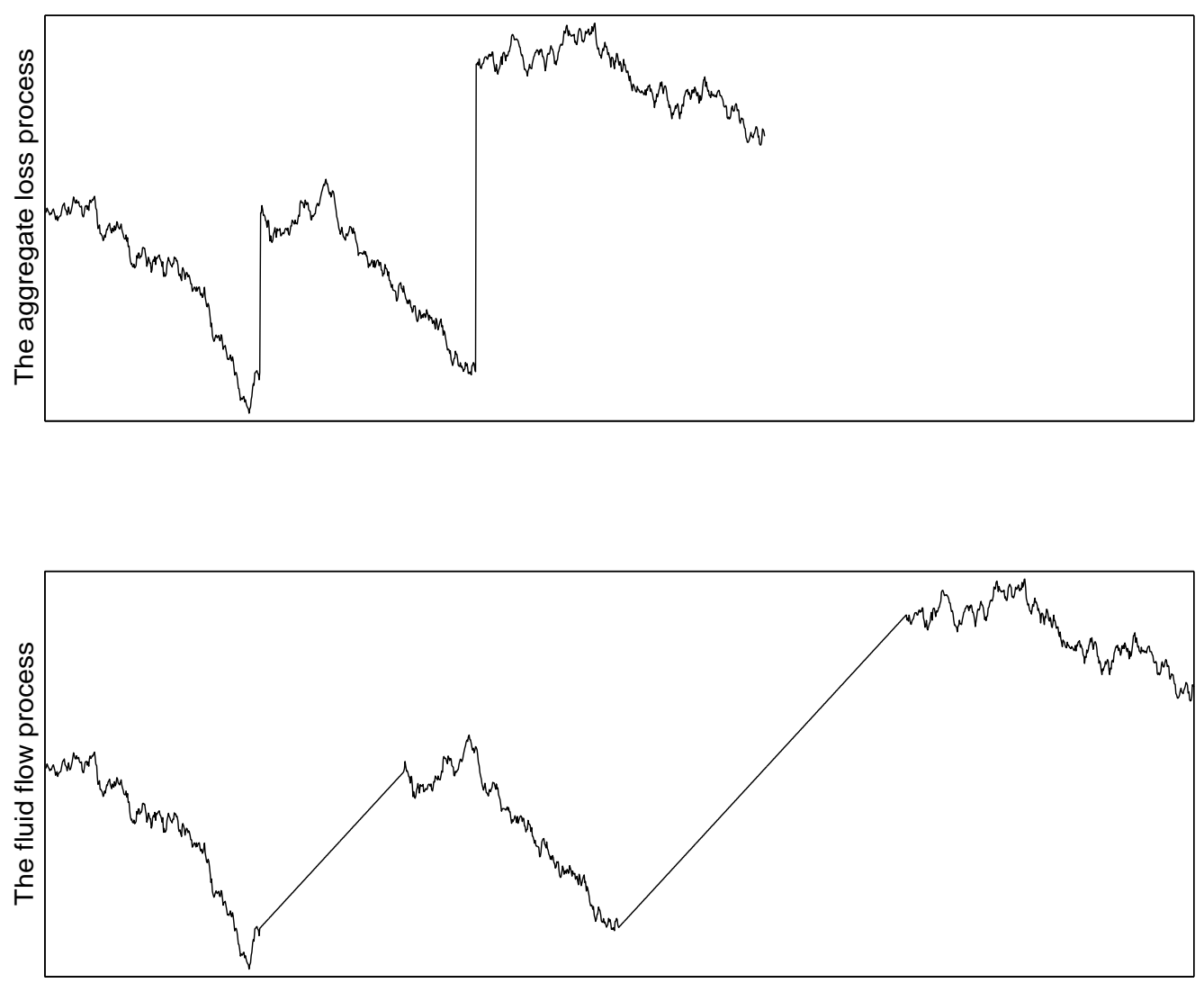

Figure 1: Sample path of perturbed aggregate loss process and partially-perturbed fluid flow

We assume that it is irreducible and has limiting distribution $\boldsymbol{\pi}=\left(\pi_{i}\right)$. The superscripts denote the dimension of the matrices. For instance, the notion for matrix $\Lambda^{(\sigma \sigma)}$ indicates that its dimension is $\left|E_{\sigma}\right| \times\left|E_{\sigma}\right|$. These conventions will be used in the sequel, and the superscript indicating the dimensions of the matrix will be omitted if they are obvious from the context.

\subsection{Illustrative Example: The Perturbed Markov Modu- lated Poisson Risk Model}

In the context of Markov modulated Poisson risk processes, we set the risk process $\left\{S_{t}\right\}_{t \geq 0}$ defined in (1) in a Markovian environment and let the premium rate, the infinitesimal variance of the Brownian motion, and the parameters of the claim amount distribution be governed by an independent Markov process $\left\{I_{t}\right\}_{t \geq 0}$ with finite state set $E_{Q}=\{1,2, \ldots, n\}$ and initial distribution $\gamma$. Its intensity matrix is denoted by $\mathbf{Q}$ which is assumed to be irreducible.

When $I_{t}=i$, the premium rate is $c_{i}$, the infinitesimal variance of the Brownian motion is $\sigma_{i}^{2}$, the arrival rate is $\lambda_{i}$ and the distribution of the claim size is $B_{i}$ 
which is phase-type distributed with representation $\left(\boldsymbol{\alpha}^{(i)}, \mathbf{T}^{(i)}\right)$ and state spaces $E^{(i)}=\left\{1^{(i)}, 2^{(i)}, \ldots, q_{i}^{(i)}\right\}$. We denote by $\mathbf{t}^{(i)}=-\mathbf{T}^{(i)} \mathbf{1}$ the absorption rates of the phase process associated with $B_{i}$, where $\mathbf{1}$ is a column vector of $1^{\prime} s$ of suitable length.

In this case, the phase set of the underlying Markov jump process $\left\{J_{t}\right\}_{t \geq 0}$ is $E=E_{\sigma} \cup E_{+}$, where $E_{\sigma}=E_{Q}$ and $E_{+}=\cup_{i} E^{(i)}$. The vector of linear drifts of the flow $\left\{\tilde{S}_{t}\right\}_{t \geq 0}$ is $\mathbf{r}=\left\{\mathbf{r}_{n}, \mathbf{r}_{p}\right\}=\left\{-c_{1},-c_{2}, \ldots,-c_{n}, 1,1, \ldots, 1\right\}$, where $\mathbf{r}_{n}=$ $\left\{-c_{1},-c_{2}, \ldots,-c_{n}\right\}$ is the vector of drifts in states $E_{\sigma}$ and $\mathbf{r}_{p}=\{1,1, \ldots, 1\}$ is the vector of drifts in states $E_{+}$. The vector of infinitesimal variances associated with the perturbation component in is states $E_{\sigma}$ is denoted by $\boldsymbol{\sigma}^{2}=\left\{\sigma_{1}^{2}, \sigma_{2}^{2}, \ldots, \sigma_{n}^{2}\right\}$.

With this setup, the infinitesimal generator of the underlying Markov jump process $\left\{J_{t}\right\}_{t \geq 0}$ is given by

$$
\boldsymbol{\Lambda}=\left(\begin{array}{cc}
\Lambda^{(\sigma \sigma)} & \Lambda^{(\sigma+)} \\
\Lambda^{(+\sigma)} & \Lambda^{(++)}
\end{array}\right)=\left(\begin{array}{cc}
\mathbf{Q}-\boldsymbol{\Delta}_{\lambda_{i}} & \boldsymbol{\Delta}_{\lambda_{i} \boldsymbol{\alpha}^{(i)}} \\
\boldsymbol{\Delta}_{\mathbf{t}^{(i)}} & \boldsymbol{\Delta}_{\mathbf{T}^{(i)}}
\end{array}\right)
$$

where $\boldsymbol{\Delta}_{\mathbf{T}^{(i)}}, \boldsymbol{\Delta}_{\lambda_{i} \boldsymbol{\alpha}^{(i)}}$ and $\boldsymbol{\Delta}_{\mathbf{t}^{(i)}}$ denote block diagonal matrices with elements $\mathbf{T}^{(i)}$, $\lambda_{i} \boldsymbol{\alpha}^{(i)}$ and $\mathbf{t}^{(i)}$ on the diagonals. We note that this generator is actually derived in Asmussen [3], page 234-236), where a mapping between the unperturbed risk process and the the fluid process was introduced. The perturbation does not change the underlining Markovian structure at all.

\section{Asmussen's Perturbed Fluid Flow Model}

We demonstrated in the previous section that for each sample path of $\left\{S_{t}\right\}_{t \geq 0}$, one can associate a unique sample path for the corresponding perturbed fluid flow process $\left\{\left(J_{t}, \tilde{S}_{t}\right)\right\}_{t \geq 0}$. Thus, we can invoke Asmussen (1995a), which presents a method to calculate the distriubution of maximal aggregate loss defined by $M=$ $\sup _{t \geq 0} \tilde{S}_{t}$. Asmussen (1995a) establishes that the existence of the maximal aggregate loss distribution is contingent upon

$$
\sum_{i \in E} \pi_{i} r_{i}<0
$$

where $E=E_{\sigma} \cup E_{+}$is the finite collection of states for the phase process $\left\{J_{t}\right\}_{t \geq 0}$, $\pi_{i}$ is the stationary probability of the embedded Markov chain $\left\{J_{t}\right\}_{t \geq 0}$ being in state $i$, and $r_{i}$ is the linear rates of increase in the fluid level while $J(t)=i$. This condition is assumed throughout this paper.

To derive the distribution of $M$, a record high phase process $\{m(x)\}_{x \geq 0}$ is defined to be the state of the underlying phase process $\left\{J_{t}\right\}_{t \geq 0}$ at the first instance that the process $\left\{\tilde{S}_{t}\right\}_{t \geq 0}$ reaches the level $x$ (see Figure 2).

Asmussen [1] then establishes that the $\{m(x)\}_{x \geq 0}$ is a terminating Markov jump process with life-length coinciding with $M$. Therefore, $M$ has a phase-type distribution whose phase generator is the sub-intensity matrix of $\{m(x)\}_{x \geq 0}$. Due to 

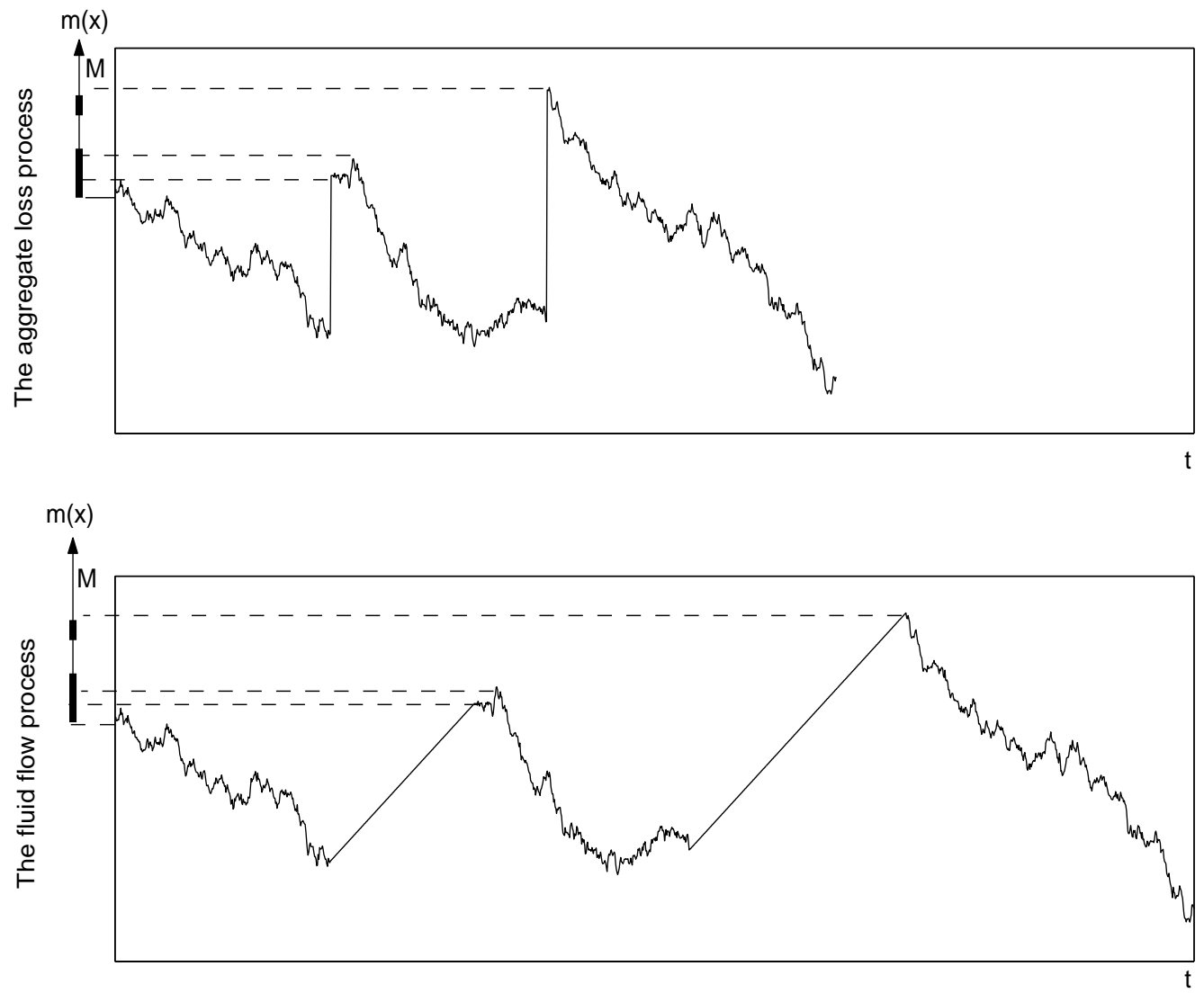

Figure 2: Sample path equivalence of the two processes, with relative maxima indicated

the property of Brownian motion, the distribution of $m(0)$ is the identical to that of $J(0)$.

Let $\mathbf{U}$ denote the sub-intensity matrix of $m(x)$. Then since ruin occurs when this maximum aggregate loss $M$ exceeds the initial surplus $u$, the probability of ruin is given by

$$
\psi(u)=\boldsymbol{\alpha} \mathrm{e}^{\mathrm{U} \cdot u} \mathbf{1}
$$

where $\boldsymbol{\alpha}$ is the initial distribution of the process $\left\{J_{t}\right\}_{t \geq 0}$.

\subsection{Determining the Matrix U}

Equation (4) demonstrates that the principle step in obtaining the ruin probability is the determination of the matrix $\mathbf{U}$. Since the state space of the process $m(x)$ is $E$, we may partition it into the block form

$$
\mathbf{U}=\left(\begin{array}{cc}
\mathbf{U}^{(\sigma \sigma)} & \mathbf{U}^{(\sigma+)} \\
\mathbf{U}^{(+\sigma)} & \mathbf{U}^{(++)}
\end{array}\right)
$$


The periods of claim payment are not subject to diffusion, so the two lower block elements of $\mathbf{U}$ are identical with their counterparts in $\boldsymbol{\Lambda}$ :

$$
\mathbf{U}^{(+\sigma)}=\Lambda^{(+\sigma)} \text { and } \mathbf{U}^{(++)}=\Lambda^{(++)} .
$$

To determine $\mathbf{U}^{(\sigma+)}$ and $\mathbf{U}^{(\sigma \sigma)}$, one must first consider what happens to the evolution of the risk process subject to diffusion during the exponentially-distributed residency time in any of the states of $E_{\sigma}$. Subsequent to this, one must determine how to piece together the total variation in the risk process during the consecutive visits to the various states in $E_{\sigma}$, between visits to states in $E_{+}$.

For the first part, Assmussen [1] in his Theorem 4.1 exploited a well-known result (see, for example, Bertoin [7]) on the distribution of the maximum increase, and the distribution of the drop from that maximum to the final level, in a Brownian motion with drift, during an exponential interval in any state $i \in E_{\sigma}$. These quantities are each exponentially distributed, independent of each other, with differing parameters $\omega_{i}>0$ and $\eta_{i}>0$. Our algorithm works with an easier result given in Theorem 4.2 in Asmussen [1]: we determine a single $\eta \geq \max _{i}\left\{\eta_{i}\right\}>0$ large enough so that the numbers $\mu_{i}>0$ and $\omega_{i}>0$ satisfy

$$
-\frac{r_{i}}{\sigma_{i}^{2}}+\sqrt{\frac{r_{i}^{2}}{\sigma_{i}^{4}}+\frac{2 \mu_{i}}{\sigma_{i}^{2}}}=\eta, \quad \omega_{i}=\frac{r_{i}}{\sigma_{i}^{2}}+\sqrt{\frac{r_{i}^{2}}{\sigma_{i}^{4}}+\frac{2 \mu_{i}}{\sigma_{i}^{2}}} .
$$

An $\eta$ that meets the foregoing requirement ensures that $\mu_{i} \geq-\Lambda_{i i}, \forall i \in E_{\sigma}$, where $\Lambda_{i i}$ denotes the $i^{t h}$ diagonal element of $\Lambda$. A further restriction is that

$$
\eta \geq-\Lambda_{i i}, \forall i \in E_{+}
$$

to ensure the adequacy of the uniformization scheme. Turning to the second part, Asmussen [1] exploits the independence of the rise and fall components of the perturbed process, and considers the conditional probability from every state $i \in E_{\sigma}$ to all other states therein, in order to establish the total amount of variation during one sojourn in $E_{\sigma}$. The net result in our terms is the following set of implicit equations for $\mathbf{U}^{(\sigma \sigma)}$ and $\mathbf{U}^{(\sigma+)}$ :

$$
\left(\begin{array}{ll}
\mathbf{U}^{(\sigma \sigma)} & \mathbf{U}^{(\sigma+)}
\end{array}\right)=\boldsymbol{\Delta}_{2 \boldsymbol{\mu} / \boldsymbol{\sigma}^{2}} \cdot\left(\begin{array}{ll}
\mathbf{Q}^{(\sigma \sigma)} & \mathbf{Q}^{(\sigma+)}
\end{array}\right) \cdot(\eta \mathbf{I}-\mathbf{U})^{-1}-\left(\begin{array}{ll}
\boldsymbol{\Delta}_{\boldsymbol{\omega}} & \mathbf{0}^{(\sigma+)}
\end{array}\right),
$$

where $\boldsymbol{\mu}$ and $\boldsymbol{\omega}$ are column vectors containing the elements $\mu_{i}$ and $\omega_{i}$ respectively, whereas $2 \boldsymbol{\mu} / \boldsymbol{\sigma}^{2}$ is a column vector with elements $2 \mu_{i} / \sigma_{i}^{2}$ and

$$
\begin{aligned}
& \mathbf{Q}^{(\sigma \sigma)}=\mathbf{I}+\boldsymbol{\Delta}_{1 / \boldsymbol{\mu}} \cdot \boldsymbol{\Lambda}^{(\sigma \sigma)}, \\
& \mathbf{Q}^{(\sigma+)}=\boldsymbol{\Delta}_{1 / \boldsymbol{\mu}} \cdot \boldsymbol{\Lambda}^{(\sigma+)} .
\end{aligned}
$$

Asmussen [1] has shown that $\mathbf{U}$ can be obtained alternatively as the result of an iterative scheme. Given matrix $\mathbf{V}$ with same dimensions as $\mathbf{U}$, we define a nonlinear matrix function $\psi_{1}$ as follows.

$$
\psi_{1}(\mathbf{V}):=\left(\begin{array}{cc}
\tilde{\mathbf{V}}^{(\sigma \sigma)} & \tilde{\mathbf{V}}^{(\sigma+)} \\
\boldsymbol{\Lambda}^{(+\sigma)} & \boldsymbol{\Lambda}^{(++)}
\end{array}\right)
$$


where $\tilde{\mathbf{V}}^{(\sigma \sigma)}$ and $\tilde{\mathbf{V}}^{(\sigma+)}$ are determined by the following, which is similar to the determination of $\mathbf{U}^{(\sigma \sigma)}$ and $\mathbf{U}^{(\sigma+)}$ in (9),

$$
\left(\begin{array}{ll}
\tilde{\mathbf{V}}^{(\sigma \sigma)} & \tilde{\mathbf{V}}^{(\sigma+)}
\end{array}\right)=\boldsymbol{\Delta}_{2 \boldsymbol{\mu} / \boldsymbol{\sigma}^{2}} \cdot\left(\begin{array}{ll}
\mathbf{Q}^{(\sigma \sigma)} & \mathbf{Q}^{(\sigma+)}
\end{array}\right) \cdot(\eta \mathbf{I}-\mathbf{V})^{-1}-\left(\begin{array}{ll}
\boldsymbol{\Delta}_{\boldsymbol{\omega}} & \mathbf{0}^{(\sigma+)}
\end{array}\right) .
$$

Then, $\mathbf{U}$ is the fixed point of the function $\psi_{1}$ and can be computed by the following iteration scheme:

$$
\mathbf{U}(n+1)=\psi_{1}(\mathbf{U}(n))
$$

with the initial value

$$
\mathbf{U}(0)=\left(\begin{array}{cc}
-\Delta_{\boldsymbol{\omega}} & \mathbf{0}^{(\sigma+)} \\
\boldsymbol{\Lambda}^{(+\sigma)} & \boldsymbol{\Lambda}^{(++)}
\end{array}\right)
$$

\section{Erlangization \& Construction of the Composite Process}

Erlangization approximates a fixed time interval of length $t$ by a random interval whose mean is $t$. If this interval comprises a fixed number $L$ of i.i.d. exponential stages, the sequence of approximations thus generated is converges to the fixed time interval as $L \rightarrow \infty$ (see, for instance, Theorem 6 of Asmussen et al. [4]).

Consider a general phase-type distribution $\operatorname{PH}(\boldsymbol{\beta}, \mathbf{H})$ for the time horizon. We construct a composite process from process $\left\{\left(J_{t}, \tilde{S}\right)\right\}_{t \geq 0}$ and $\operatorname{PH}(\boldsymbol{\beta}, \mathbf{H})$. Let $\left\{J_{t}^{H}\right\}_{t \geq 0}$ denote the underlying Markov chain for the phase-type distribution $\operatorname{PH}(\boldsymbol{\beta}, \mathbf{H})$, and denote its state space by $E_{H}$ with order $p_{H}$. To obtain the composite process, denoted by $\left\{\left(J_{t}^{H}, J_{t}\right)\right\}_{t \geq 0}$ we pair each of the phases of the "horizon" $\operatorname{PH}(\boldsymbol{\beta}, \mathbf{H})$ with its concurrent phase $i$ for $\left\{J_{t}\right\}_{t \geq 0}$ at time $t$. The resulting Markov process has state space $E=E_{0} \cup E_{H_{\sigma}} \cup E_{H_{+}}$, where $E_{0}=\{C\}$ for the absorbing state once the horizon has been reached ( $C$ here denoting "cemetary"), $E_{H_{\sigma}}=E_{H} \times E_{\sigma}$ and $E_{H_{+}}=E_{H} \times E_{+}$. Working with this construction, the probability of an event of interest occurring by time $t$ can be approximated by that the probability of the event occurring while the composite process resides among the transient states.

When $J_{t}^{H}=i \in E_{H}, J_{t}=j \in E_{\sigma},\left\{\tilde{S}_{t}\right\}_{t \geq 0}$ behaves like a Brownian motion with negative drift $c_{j}$ and infinitesimal variance $\sigma_{j}^{2}$. In contrast, when $J_{t}=j \in E_{+}$ it increases at unit rate regardless of the value of $J_{t}^{H}$. The vectors of drifts and infinitesimal variances of the fluid process $\left\{\tilde{S}_{t}\right\}_{t \geq 0}$ in all possible states of $\left\{\left(J_{t}^{H}, J_{t}\right)\right\}$ are given by, respectively

$$
\mathbf{r}_{H}=\mathbf{1}_{H} \otimes \mathbf{r} ; \quad \boldsymbol{\sigma}_{H}^{2}=\mathbf{1}_{H} \otimes \boldsymbol{\sigma}^{2}
$$

where $\otimes$ represents the Kronecker product and the vectors $r$ and $\boldsymbol{\sigma}^{\mathbf{2}}$ were defined in section 2.1 .

It is obvious that the process $\left\{\left(J_{t}^{H}, J_{t}\right)\right\}_{t \geq 0}$ has initial distribution $(0 \boldsymbol{\beta} \otimes \boldsymbol{\alpha})$. While $J_{t} \in E_{\sigma}$, it is possible for $J_{t}^{H}$ to change state. In contrast, when $J_{t} \in E_{+}$, there is no passage of real time and consequently, the current value of $J_{t}^{H}$ among 
the transient stages must be preserved. Therefore, the generator of the precess $\left\{\left(J_{t}^{H}, J_{t}\right)\right\}_{t \geq 0}$ is given by

$$
\left(\begin{array}{ccc}
0 & \mathbf{0} & \mathbf{0} \\
\mathbf{h} \otimes \mathbf{e} & \boldsymbol{\Lambda}_{H}^{\left(H_{\sigma \sigma}\right)} & \boldsymbol{\Lambda}_{H}^{\left(H_{\sigma+}\right)} \\
\mathbf{0} & \boldsymbol{\Lambda}_{H}^{\left(H_{+\sigma}\right)} & \boldsymbol{\Lambda}_{H}^{\left(H_{++}\right)}
\end{array}\right)=\left(\begin{array}{ccc}
0 & \mathbf{0} & \mathbf{0} \\
\mathbf{h} \otimes \mathbf{e} & \mathbf{H} \oplus \boldsymbol{\Lambda}^{(\sigma \sigma)} & \mathbf{I}_{H} \otimes \boldsymbol{\Lambda}^{(\sigma+)} \\
\mathbf{0} & \mathbf{I}_{H} \otimes \boldsymbol{\Lambda}^{(+\sigma)} & \mathbf{I}_{H} \otimes \boldsymbol{\Lambda}^{(++)}
\end{array}\right)
$$

where $\oplus$ represents the Kronecker sum, and $\mathbf{h}=-\mathbf{H} \mathbf{1}$. Since we are only concerned with the evolution of this process prior to absorption, we pick out the four blocks from south east corner of the above matrix and write the resultant subintensity matrix as

$$
\boldsymbol{\Lambda}_{H}=\left(\begin{array}{cc}
\Lambda_{H}^{\left(H_{\sigma \sigma}\right)} & \Lambda_{H}^{\left(H_{\sigma+}\right)} \\
\Lambda_{H}^{\left(H_{+\sigma}\right)} & \Lambda_{H}^{\left(H_{++}\right)}
\end{array}\right)
$$

In the special case where $\mathrm{PH}(\boldsymbol{\beta}, \mathbf{H})$ is of order 1 , the time horizon is exponential with rate $a$ (say), we have

$$
\boldsymbol{\Lambda}_{H}=\left(\begin{array}{cc}
\Lambda^{(\sigma \sigma)}-a \mathbf{I} & \boldsymbol{\Lambda}^{(\sigma+)} \\
\Lambda^{(+\sigma)} & \boldsymbol{\Lambda}^{(++)}
\end{array}\right) .
$$

\section{The Ruin Probability Prior to a Phase-Type Time Horizon}

Similar to section 3, we define the record high phase process $\left\{m_{H}(x)\right\}_{x \geq 0}$ to be the state of the underlying phase process $\left\{\left(J_{t}^{H}, J_{t}\right)\right\}_{t \geq 0}$ at the first instance that the process $\left\{\tilde{S}_{t}\right\}_{t \geq 0}$ is at the maximal value $x$. Then $\left\{m_{H}(x)\right\}$ is a terminating Markov process whose generator can be partitioned into

$$
\mathbf{U}_{H}=\left(\begin{array}{cc}
\mathbf{U}_{H}^{\left(H_{\sigma \sigma}\right)} & \mathbf{U}_{H}^{\left(H_{\sigma+}\right)} \\
\mathbf{U}_{H}^{\left(H_{+\sigma}\right)} & \mathbf{U}_{H}^{\left(H_{++}\right)}
\end{array}\right) .
$$

Once $\mathbf{U}_{H}$ has been obtained, the probability of ruin before time horizon $\mathrm{PH}(\boldsymbol{\beta}, \mathbf{H})$ is given by

$$
\psi(u, \mathbf{H})=(\boldsymbol{\beta} \otimes \boldsymbol{\alpha}) \mathrm{e}^{u \cdot \mathbf{U}_{H}} \mathbf{1} .
$$

As in Dufrense and Gerber [10], we can decompose the ruin probability by its cause. The probability of ruin due to diffusion is given by

$$
\psi(u, \mathbf{H})=(\boldsymbol{\beta} \otimes \boldsymbol{\alpha}) \mathrm{e}^{u \cdot \mathbf{U}_{H}}\left(\begin{array}{c}
\mathbf{1}^{(\sigma)} \\
\mathbf{0}^{(+)}
\end{array}\right)
$$


while the probability of ruin due to claims is given by

$$
\psi(u, \mathbf{H})=(\boldsymbol{\beta} \otimes \boldsymbol{\alpha}) \mathrm{e}^{u \cdot \mathbf{U}_{H}}\left(\begin{array}{c}
\mathbf{0}^{(\sigma)} \\
\mathbf{1}^{(+)}
\end{array}\right) .
$$

Our approach for determining the matrix $\mathbf{U}_{H}$ follows the same steps described in Section 3.1, now applied to the composite process. Specifically, by substituting parameters $\mathbf{r}_{H}$ and $\boldsymbol{\sigma}_{H}$ into formula (7), and ensuring that the resulting $\eta_{H}$ satisfies (8) for rate matrix $\boldsymbol{\Lambda}_{H}$ given in (14), we obtain the vectors $\boldsymbol{\mu}_{H}$ and $\boldsymbol{\omega}_{H}$ which are used for computing $\mathbf{U}_{H}$. It is easily seen that $\boldsymbol{\mu}_{H}$ and $\boldsymbol{\omega}_{H}$ have the same structures as those of $\mathbf{r}_{H}$ and $\boldsymbol{\sigma}_{H}$, which we choose to express as

$$
\boldsymbol{\mu}_{H}=\mathbf{1}_{H} \otimes \boldsymbol{\mu}^{\prime}, \quad \boldsymbol{\omega}_{H}=\mathbf{1}_{H} \otimes \boldsymbol{\omega}^{\prime} .
$$

where $\mathbf{1}_{H}$ is a column vector of ones, while $\boldsymbol{\mu}^{\prime}$ and $\boldsymbol{\omega}^{\prime}$ are appropriately dimensioned column vectors containing the individual elements $\mu_{i}^{\prime}, \omega_{i}^{\prime}$.

Then, the direct application of the algorithm presented in section 3.1 to the matrix $\mathbf{U}_{H}$ yields the following nonlinear matrix equation:

$$
\mathbf{U}_{H}=\psi_{2}\left(\mathbf{U}_{H}\right):=\left(\begin{array}{cc}
\mathbf{V}_{H}^{\left(H_{\sigma \sigma}\right)} & \mathbf{V}_{H}^{\left(H_{\sigma+}\right)} \\
\boldsymbol{\Lambda}_{H}^{\left(H_{+\sigma}\right)} & \boldsymbol{\Lambda}_{H}^{\left(H_{++}\right)}
\end{array}\right)
$$

where matrices $\mathbf{V}_{H}^{\left(H_{\sigma \sigma}\right)}$ and $\mathbf{V}_{H}^{\left(H_{\sigma+}\right)}$ are determined by the following equation

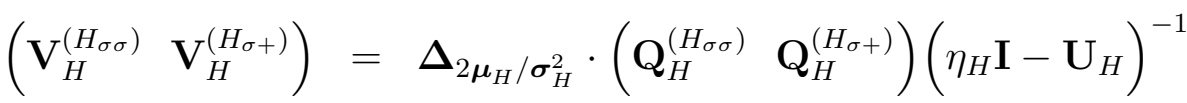

$$
\begin{aligned}
& -\left(\begin{array}{ll}
\Delta_{\omega_{H}} & 0^{\left(H_{\sigma+}\right)}
\end{array}\right)
\end{aligned}
$$

and where

$$
\begin{aligned}
& \mathbf{Q}_{H}^{\left(H_{\sigma \sigma}\right)}=\mathbf{I}+\boldsymbol{\Delta}_{1 / \boldsymbol{\mu}_{H}} \cdot \boldsymbol{\Lambda}^{\left(H_{\sigma \sigma}\right)}=\mathbf{I}+\mathbf{H} \otimes \boldsymbol{\Delta}_{1 / \boldsymbol{\mu}^{\prime}}+\mathbf{I}_{H} \otimes\left(\boldsymbol{\Delta}_{1 / \boldsymbol{\mu}^{\prime}} \cdot \boldsymbol{\Lambda}^{(\sigma \sigma)}\right), \\
& \mathbf{Q}_{H}^{\left(H_{\sigma+}\right)}=\boldsymbol{\Delta}_{1 / \boldsymbol{\mu}_{H}} \cdot \boldsymbol{\Lambda}^{\left(H_{\sigma+}\right)}=\mathbf{I}_{H} \otimes\left(\boldsymbol{\Delta}_{1 / \boldsymbol{\mu}^{\prime}} \cdot \boldsymbol{\Lambda}^{(\sigma+)}\right) .
\end{aligned}
$$

In addition, $\mathbf{U}_{H}$ can be computed by the following iteration scheme:

$$
\mathbf{U}_{H}(n+1)=\psi_{2}\left(\mathbf{U}_{H}(n)\right),
$$

where the initial value $\mathbf{U}_{H}(0)$ is given by

$$
\mathbf{U}_{H}(0)=\left(\begin{array}{cc}
-\boldsymbol{\Delta}_{\boldsymbol{\omega}_{H}}^{\left(H_{\sigma \sigma}\right)} & \mathbf{0}^{\left(H_{\sigma+}\right)} \\
\boldsymbol{\Lambda}_{H}^{\left(H_{+\sigma}\right)} & \boldsymbol{\Lambda}_{H}^{\left(H_{++}\right)}
\end{array}\right)
$$




\section{Simplifications for Erlang Horizons}

One problem with the formulas of the last section is that the size of the matrix $\mathbf{U}_{H}$ grows quickly as the Horizon matrix $H$ does. However, with an Erlang horizon, the $\mathbf{U}_{H}$ possesses a special structure, since the process can only move forward one stage at a time, and can never move backwards. This yields a $\mathbf{U}_{H}$ that is block upper triangular in form, with repeating block elements along the successive diagonals. This structure was noted first by Asmussen et al. [4] for the classical risk model to approximate the finite time ruin probability, and their paper derived a recursive formula to calculate the entries.

We define "block Toeplitz structure" as a block upper-triangular matrix such that $\forall i \leq j$, the $(i, j)$ th block is the same for all blocks with the same index $j-i$. Thus the main diagonal blocks, superdiagonal blocks, and so on, each feature a single, repeating block.

Our matrix $\mathbf{U}_{H}$ possesses this structure as well, and the latter part of this section presents the equations to obtain the successive blocks. As a result, rather than calculating one very large matrix, it suffices to compute $L$ smaller block elements of $\mathbf{U}_{H}$ that repeat, and therefore the computational expense is greatly reduced. Recently, Ramaswami et al. [13] extend these results to a general fluid flow model with Erlang horizon incorporated. In this section, we further extend these results to the perturbed fluid flow model.

\subsection{The Structure of the Matrix $\mathbf{U}_{H}$}

Theorem 1 When the horizon has Erlang distribution, the block matrices $\boldsymbol{U}_{H}^{\left(H_{\sigma \sigma}\right)}$ and $\boldsymbol{U}_{H}^{\left(H_{\sigma+}\right)}$ in $\boldsymbol{U}_{H}$ have $L \times L$ block Toeplitz structures. That is,

$$
\begin{aligned}
\boldsymbol{U}_{H}^{\left(H_{\sigma \sigma}\right)}= & \left(\begin{array}{ccccc}
\boldsymbol{U}_{0}^{(\sigma \sigma)} & \boldsymbol{U}_{1}^{(\sigma \sigma)} & \ldots & \boldsymbol{U}_{L-2}^{(\sigma \sigma)} & \boldsymbol{U}_{L-1}^{(\sigma \sigma)} \\
\boldsymbol{O} & \boldsymbol{U}_{0}^{(\sigma \sigma)} & \ldots & \boldsymbol{U}_{L-3}^{(\sigma \sigma)} & \boldsymbol{U}_{L-2}^{(\sigma \sigma)} \\
\ldots & \ldots & \ldots & \ldots & \ldots \\
\boldsymbol{O} & \boldsymbol{O} & \ldots & \boldsymbol{U}_{0}^{(\sigma \sigma)} & \boldsymbol{U}_{1}^{(\sigma \sigma)} \\
\boldsymbol{O} & \boldsymbol{O} & \ldots & \boldsymbol{O} & \boldsymbol{U}_{0}^{(\sigma \sigma)}
\end{array}\right) \text { and } \\
\boldsymbol{U}_{H}^{\left(H_{\sigma+}\right)} & =\left(\begin{array}{ccccc}
\boldsymbol{U}_{0}^{(\sigma+)} & \boldsymbol{U}_{1}^{(\sigma+)} & \ldots & \boldsymbol{U}_{L-2}^{(\sigma+)} & \boldsymbol{U}_{L-1}^{(\sigma+)} \\
\boldsymbol{O} & \boldsymbol{U}_{0}^{(\sigma+)} & \ldots & \boldsymbol{U}_{L-3}^{(\sigma+)} & \boldsymbol{U}_{L-2}^{(\sigma+)} \\
\ldots & \ldots & \ldots & \ldots & \ldots \\
\boldsymbol{O} & \boldsymbol{O} & \ldots & \boldsymbol{U}_{0}^{(\sigma+)} & \boldsymbol{U}_{1}^{(\sigma+)} \\
\boldsymbol{O} & \boldsymbol{O} & \ldots & \boldsymbol{O} & \boldsymbol{U}_{0}^{(\sigma+)}
\end{array}\right)
\end{aligned}
$$

where $\boldsymbol{U}_{i}^{(\sigma \sigma)}$ and $\boldsymbol{U}_{i}^{(\sigma+)}$ are matrices with dimensions $\left|E_{\sigma}\right| \times\left|E_{\sigma}\right|$ and $\left|E_{\sigma}\right| \times\left|E_{+}\right|$ respectively. 
Table 1: Finite ruin probabilities by Erlangian Approximation $(t=100, u=10)$, $\sigma=\{10,1\}$ (diffusion case)

\begin{tabular}{|c||c|c||c|c|}
\hline $\mathrm{L}$ & non-diffusion & Richardson & diffusion & Richardson \\
\hline \hline 1 & 0.572 & & 0.898 & \\
3 & 0.609 & 0.627 & 0.925 & 0.938 \\
5 & 0.617 & 0.630 & 0.928 & 0.933 \\
7 & 0.621 & 0.630 & 0.930 & 0.933 \\
10 & 0.624 & 0.630 & 0.931 & 0.933 \\
15 & 0.626 & 0.630 & 0.931 & 0.933 \\
20 & 0.627 & 0.630 & 0.932 & 0.933 \\
25 & 0.627 & 0.630 & 0.932 & 0.933 \\
30 & 0.628 & 0.630 & 0.932 & 0.933 \\
\hline
\end{tabular}

The proof of the block Toeplitz structure and recursive algorithms for the efficient computation of the block elements appear in the companion paper to this work-inprogress. The full paper has been submitted for publication just recently.

\section{$7 \quad$ Examples}

Example 1. We consider the "Contagion" example in Badescu et al [6], in which the system switches between two environmental conditions. Environment A corresponds to a normal situation, while environment $\mathrm{B}$ reflects periods of contagion, with an extra stream of claims due to disease. The environment switches from $A$ to $B$ at rate $\alpha_{A}$ and from $B$ to $A$ at rate $\alpha_{B}$.

We use two sets of variance parameters: $\sigma=\{10,1\}$ for the real diffusion case, and $\sigma=\{0.01,0.01\}$ to approximate the non-diffusion case. As in Asmussen et. al [4] and Stanford et al [14] we improve the estimate with the Richardson extrapolation

$$
\psi_{L+k}(u, T) \approx \frac{(L+k) \psi\left(u, H_{L+k}\right)-L \psi\left(u, H_{L}\right)}{k},
$$

where $T$ is the fixed time, $L+k$ and $L$ are stages of Erlang horizons.

We calculated the probability of ruin before time $t=100$ for an initial surplus $u=10$. We compared our results to those in Badescu et al [6] for the non-diffusion case, and Badescu \& Breuer [5] for the diffusion case; the results agree to 3 decimal places with the limiting value in the columns denoted "Richardson". In both cases, the approximation is already quite reasonable for even small values of $L$, but the results improve much more quickly by making use of the Richardson extrapolation.

Figure 3 displays the total ruin probability and its components as a function of the infinitesimal variance, for $u=10$ and $L=2$. Obviously, as $\sigma$ gets bigger, both the total probability of ruin and the probability of ruin due to diffusion increase. 
Table 2: Finite ruin probabilities by Erlangian Approximation $(t=1000, u=100)$

\begin{tabular}{cccccc}
\hline$\sigma$ & $L=1$ & $L=3$ & $L=5$ & $L=7$ & $L=9$ \\
\hline \hline$\{4.0,4.0\}$ & 0.368 & 0.427 & 0.442 & 0.448 & 0.452 \\
$\{3.0,3.0\}$ & 0.315 & 0.365 & 0.378 & 0.384 & 0.387 \\
$\{2.5,2.5\}$ & 0.292 & 0.338 & 0.350 & 0.355 & 0.358 \\
$\{2.0,2.0\}$ & 0.273 & 0.315 & 0.325 & 0.330 & 0.333 \\
$\{1.5,1.5\}$ & 0.258 & 0.297 & 0.306 & 0.311 & 0.313 \\
$\{1.0,1.0\}$ & 0.248 & 0.284 & 0.293 & 0.297 & 0.299 \\
$\{0.2,0.2\}$ & 0.240 & 0.274 & 0.283 & 0.287 & 0.289 \\
$\{0.1,0.1\}$ & 0.239 & 0.274 & 0.282 & 0.286 & 0.288 \\
$\{0.01,0.01\}$ & 0.239 & 0.273 & 0.282 & 0.286 & 0.288 \\
\hline
\end{tabular}

Example 2. This example considers the Sparre-Andersen model presented in Thorin \& Wikstad [17], Table 8. We calculated the ruin probability for different values of $\sigma$. The results are listed in Table 3, which shows that the ruin probability increases as the infinitesimal variance increases. When $\sigma=0.01$, the ruin probability is identical to 3 decimals to that obtained by Stanford et al [14].

\section{Acknowledgements}

This work has been supported by the Natural Sciences and Engineering Research Council of Canada (NSERC), through the Discovery grants of Drs. Ren and Stanford, and the NSERC postgraduate scholarship of Mr. Yu (Ph. D. candidate). 


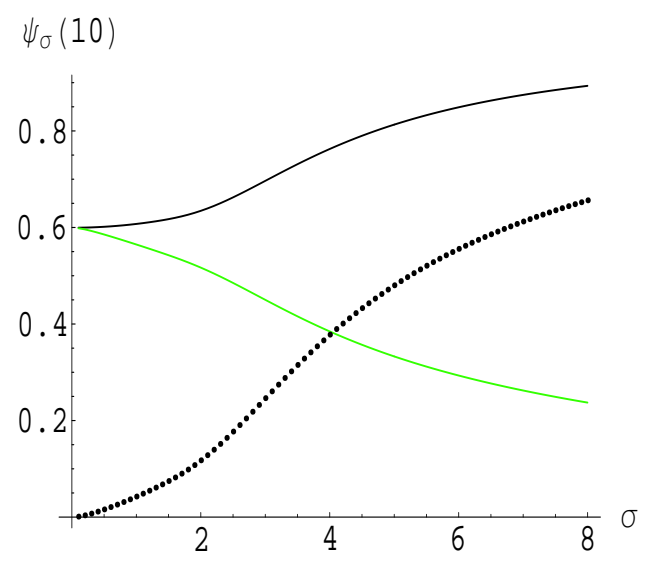

Figure 3: Decomposition of ruin probabilities. Solid: total ruin probability; Light solid: probability of ruin due to claims; Dashed: probability of ruin due to diffusion

\section{References}

[1] Asmussen, S. (1995a) Stationary distributions for fluid flow models with or without Brownian noise. Commum. Statist. - Stochastic Models 11 21-49.

[2] Asmussen, S. (1995b) Stationary distributions via first passage times. Advances in Queueing: Models, Methods 6 Problems (Dshalalow, J ed.), 79-102. CRC Press, Boca Raton, Florida.

[3] Asmussen, S. (2000) Ruin Probabilities. World Scientific.

[4] Asmussen, S., Avram, F. And Usabel, M. (2002) Erlangian approximations for finite-horizon ruin probabilities. Astin Bulletin 32 267-281.

[5] Badescu, A. And Breuer, L. (2008) The use of vector-valued martingles in risk theory. Accepted for publication in Blätter.

[6] Badescu, A., Breuer, L., Da Silva Soares, A., Latouche, G., Remiche, M.A. And Stanford, D.A. (2005) Risk processes analyzed as fluid queues. Scandinavian Actuarial Journal, 2, 127-141.

[7] Bertoin, J. (1997) Exponential decay and ergodicity of completely asymmetric Lévy Processes in a finite interval. Annals of Applied Probability 7, 156-169.

[8] Dickson, D.C.M And Waters, H.R. (1991) Recursive calculation of survival probabilities. Astin Bulletin, 22, 199-221.

[9] Drekic, S. and Willmot, G.E. (2003) On the density and moments of the time of ruin with exponential claims. Astin Bulletin, 33, 11-21. 
[10] Dufresne, F. And Gerber, H.U. (1991) Risk theory for the compound Poisson process that is perturbed by diffusion. Insurance: Mathematics and Economics, 10, 51-59.

[11] Li, S. And Garrido, J. (2005) The Gerber-Shiu function in a Sparre Andersen risk process perturbed by diffusion. Scandinavian Actuarial Journal 3, 161-186.

[12] Lu, Y. AND Tsai, C. (2007). The expected discounted penalty at ruin fpr a Markov-modulated risk process perturbed by diffusion. North American Actuarial Journal 11, 136-152.

[13] Ramaswami, V., Woolford, D.G. \& Stanford, D.A. The Erlangization method for Markovian fluid flows.

[14] Stanford, D.A., Avram, F., Badescu, A., Breuer, L., Da Silva Soares, A. And Latouche, G. (2005) Phase-type approximations to finitetime ruin probabilities in the Sparre-Andersen and stationary renewal risk models. Astin Bulletin 35, 131-144.

[15] Stanford, D.A. And Stroiński, K.J. (1994) Recursive method for computing finite-time ruin probabilies for phase-distributed claim sizes. Astin Bulletin, 24, 235-254.

[16] Stanford, D.A., Stroiński, K.J. and Lee, K. (2000) Ruin probabilities based at claim instants for some non-Poisson claim processes. Insurance: Mathematics and Economics 26, 251-267.

[17] Thorin, O. and Wikstad, N. (1973) Numerical evaluation of ruin probabilities. Astin Bull. VII 137-153.

[18] Thorin, O. and Wikstad, N. (1977) Calculation of ruin probabilities when the claim distribution is lognormal. Astin Bull. IX 231-246.

[19] Tsai, C.C.-L. And Willmot, G.E. (2002) A generalized defective renewal equation for the surplus process perturbed by diffusion. Insurance: Mathematics and Economics 30, 51-66. 